of EN regimen on gut microbiome composition. 1) Quince, Am J Gastroenterol 110, 1718-1729; 2) Logan, APT 50, 664-674, 2019; 3) Gupta, IBD 19, 1374-1378, 2013; 4) Lee, IBD 21, 1786-1793, 2015

\section{RATE AND SEVERITY OF 30-DAY AND 1-YEAR COMPLICATIONS ARISING FROM PERCUTANEOUS ENDOSCOPIC GASTROSTOMY USE}

${ }^{1}$ Conor Boylan*, ${ }^{2}$ Diane Barrett, ${ }^{2}$ Vincent Li, ${ }^{2}$ Leanne Dean, ${ }^{2}$ Sue Merrick, ${ }^{2}$ Aravinth Murugananthan, ${ }^{2,3}$ Helen Steed. 'University Of Birmingham, Birmingham, UK; ${ }^{2}$ Royal Wolverhampton NHS Trust, Wolverhampton, UK; ${ }^{3}$ University of Wolverhampton, Wolverhampton, UK

\subsection{6/gutjnl-2020-bsgcampus.48}

Introduction Percutaneous Endoscopic Gastrostomy (PEG) feeding is utilised in patients with exceptionally poor oral intake, such as those undergoing treatment for head and neck cancer or dysphagia. PEG feeding increases the risk of many complications which are important in pre-insertion counselling and post-insertion management. While some papers have been published on this topic, this is the first UK study to review longitudinal gastrostomy complications since the 2004 NCEPOD audit of PEG deaths and the subsequent changes in practice.

Methods Single-centre retrospective chart review of all patients receiving PEG insertion between January 2016 and December 2018. Subgroup analysis compared those who were cared for with professional help vs those who relied on self/family support using chi-squared and Fisher exact analysis.

Results 306 patients met the inclusion criteria. The mean age at insertion was 67 years. The majority were cared for in their own home $(80.4 \%)$ by themselves or family $(74.8 \%)$. 127 were inserted for dysphagia and 165 prophylactically prior to treatment for head and neck cancer.

$16.7 \%$ had a complication in the first 30 days. The most common complication was pain (45.3\%), followed by a weeping/irritated site $(17.2 \%)$, leaking tube $(6.3 \%)$ and site infection (6.3\%). $50.0 \%$ of 30-day complications were 'mild' (treated in the community), 48.4\% were 'moderate' (reviewed in secondary care) and $1.6 \%$ were 'severe' (required an invasive procedure to rectify).

$35.6 \%$ experienced at least one complication in the first year. The most common was pain (27.6\%), followed by a weeping/irritated site (17.8\%), external overgranuation (11.4\%) and site infection (11.4\%). 53.0\% were 'mild', 40.5\% 'moderate' and $6.5 \%$ 'severe'.

The incidence of serious gastric bleeds over the 1-year period was $2.2 \%$, aspiration pneumonia occurred in 3 patients $(1.6 \%)$ and buried bumper syndrome (BBS) occurred in 1 (0.6\%). 30-day mortality for patients post-insertion was $4.2 \%$, with the 10/13 of these falling into the dysphagia group.

Subgroup analysis showed those who relied on self- or family-care had a $63 \%$ higher chance of developing at least one complication over a 1-year period compared to those with professional support. This was statistically significant at $\mathrm{p}=$ 0.0177 .

Conclusions This study represents one of the largest of its kind evaluating the complications arising from PEG insertion. The findings correlate reasonably well with published data.

This study provides valuable data on the rate and severity of complications arising from PEG use and has implications for consenting and counselling patients pre-insertion as well as planning support services and post-insertion management.

\section{O49 DIETARY TRIGGERS OF COLONIC INFLAMMATION FOLLOWING EXCLUSIVE ENTERAL NUTRITION TREATMENT IN CHILDREN WITH CROHN'S DISEASE}

${ }^{1}$ Konstantinos Gkikas*, ${ }^{1}$ Michael Logan, ${ }^{1}$ Ben Nichols, ${ }^{1}$ Clare M Clark, ${ }^{1}$ Umer Z ljaz, ${ }^{2}$ Lisa Gervais, ${ }^{2}$ Hazel Duncan, ${ }^{2}$ Vikki Garrick, ${ }^{2}$ Lee Curtis, ${ }^{2}$ Elaine Buchanan, ${ }^{2}$ Tracey Cardigan, ${ }^{3}$ Lawrence Armstrong, ${ }^{4}$ Caroline Delahunty, ${ }^{2}$ Diana M Flynn, ${ }^{2}$ Andrew R Barclay, ${ }^{2}$ Rachel Tayler, ${ }^{2}$ Richard Hansen, ${ }^{2}$ Richard K Russell, ${ }^{1}$ Konstantinos Gerasimidis. 'University Of Glasgow, Glasgow, UK; ${ }^{2}$ Royal Hospital for Children, Glasgow, UK;

${ }^{3}$ Crosshouse Hospital, Kilmarnock, UK; ${ }^{4}$ Wishaw General Hospital, Wishaw, UK

\subsection{6/gutjnl-2020-bsgcampus.49}

Introduction Exclusive enteral nutrition (EEN) ameliorates gut inflammation in children with Crohn's disease (CD). We have previously described the rapid rise in faecal calprotectin levels (FC) when children with CD return to their habitual diet after
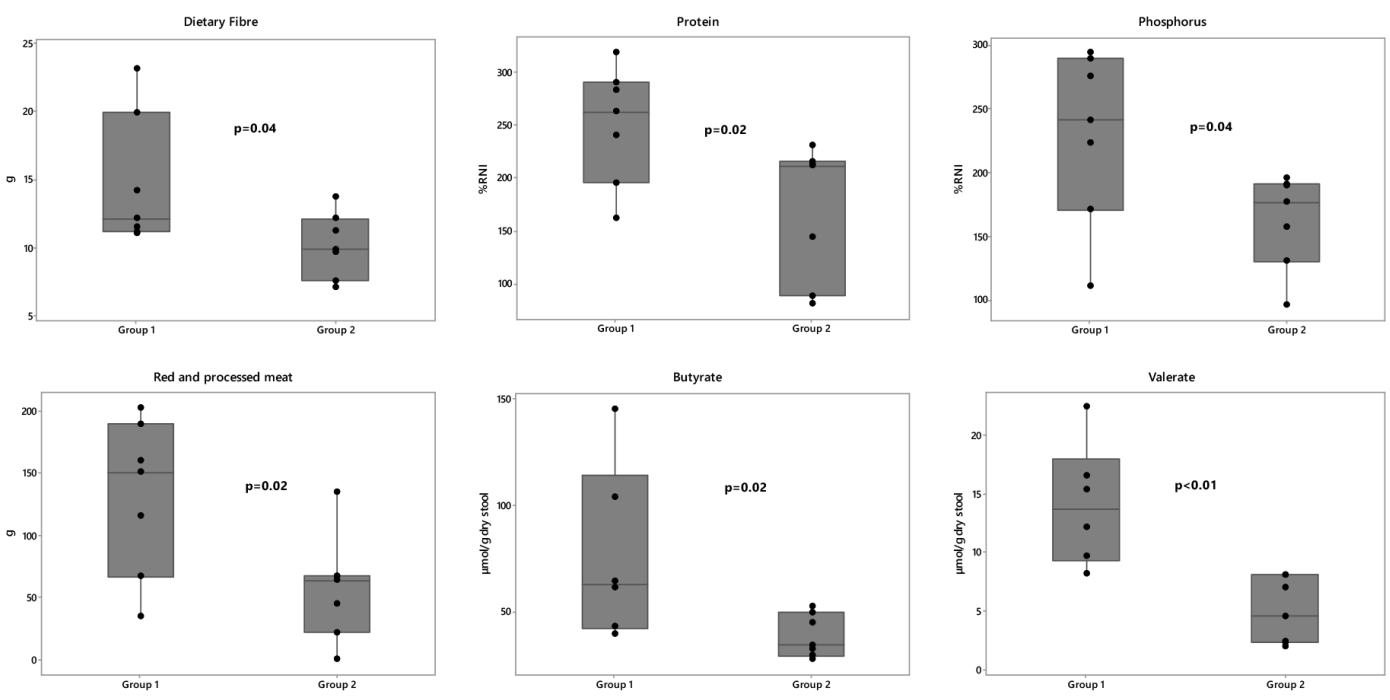

Abstract 049 Figure 1 Comparisons of intake of nutrients and foods and Short chain fatty acid levels between Group 1 (above median FC) and Group 2 (below median FC) in children with Crohn's disease, at food reintroduction, post EEN completion. 
EEN treatment. ${ }^{1}$ We explored dietary triggers of CD relapse by performing dietary assessment and measuring faecal food biomarkers in children with CD during early food reintroduction.

Methods Combination of clinical remission post EEN (weighted Paediatric Crohn's Disease Activity Index <12.5) and a significant drop in FC (>500 mg/kg/>35\%), was used to define the patient group. All patients provided 3-day estimated weight food diaries and a faecal sample. Patients were divided equally for statistical analysis purposes in two groups; above (Group 1) and below (Group 2) the median FC concentration at food reintroduction [900 mg/kg (341, 1,243)]. Nutrient and food group analysis was performed with WinDiets 10. Faecal short chain fatty acids, gluten immunogenic peptide and starch were measured as proxy of fibre, gluten and malabsorbed/resistant starch.

Results 14 children provided a FC sample within $21(15,51)$ days post EEN. Classification of patients in the two groups resulted in significantly different FC values: Group 1: 1181 $\mathrm{mg} / \mathrm{kg}$ (1024, 1781) vs Group 2: $411 \mathrm{mg} / \mathrm{kg}$ (130, 651) $(\mathrm{p}<0.01)$. Total energy intake did not differ between the two groups $(\mathrm{p}=0.37)$. Patients in Group 1 consumed more fibre, protein and phosphorus than Group $2(p=0.04, p=0.02$, $\mathrm{p}=0.04)$. Butyrate and valerate levels were higher in Group 1 than Group $2(\mathrm{p}=0.02, \mathrm{p}<0.01)$, whereas no differences were observed in GIP and faecal starch levels. Patients in Group 1 reported higher red $\&$ processed meat consumption $(p=0.02)$. Conclusions This analysis highlights dietary components potentially associated with recurrence of colonic inflammation in children with CD during early food reintroduction. These findings should be confirmed in larger studies.

\section{REFERENCE}

1. Logan, M. et al. 2019 AP\&T 50:664-674.

\section{LONGITUDINAL ANALYSIS OF THE METABOLOME IN EARLY LIFE IN PRETERM BABIES}

${ }^{1}$ Alessandra Frau, ${ }^{1}$ Lauren Lett, ${ }^{2}$ Janet Berrington, ${ }^{1}$ Rachael Slater, ${ }^{1}$ David Hughes, ${ }^{2}$ Nicholas Embleton, ${ }^{1}$ Chris Probert*. ${ }^{1}$ University Of Liverpool, Liverpool, UK; ${ }^{2}$ Newcastle Hospitals NHS Trust, Newcastle upon Tyne, UK

\subsection{6/gutjnl-2020-bsgcampus. 50}

Background Information on the metabolome in early life is still limited. Here, we describe the evolution of the metabolome in preterm babies during their first weeks of life.

Methods Multiple stool samples from 69 preterm babies were collected across 7 hospitals in the UK. Volatile organic compounds (VOCs) were analysed by running an aliquot of stool (mean $=80.6 \mathrm{mg}, \mathrm{SD}=12.3 \mathrm{mg}$ ) gas chromatography $/$ mass spectrometry. Data were interpreted using AMDIS with NIST reference library. Statistical analysis to identify factors that influenced the metabolome included clustering, PERMANOVA and linear mixed-effects modeling were carried out in $\mathrm{R}$.

Results Several factors influenced the metabolomic results; the most relevant, was as expected, the individual, followed by the hospital, the babies age in days, and the gestational age. As samples were run in two different batches, a batch effect was observed. The age of the baby influenced both the number of VOCs and the VOC profile. Number of VOCs increased over time; a sharp rise was observed after day 5 and it was stablised after day 10. In terms of VOCs observed, a shift was observed after day 5, with the observation of
VOCs produced during, or by-products of carbohydrate, fermentation (i.e. butane-2,3-dione, propyl acetate, propan-1-ol and propyl propanoate). Meanwhile, branched short chain fatty acid (BSCFA), produced by bacteria in the colon during branched amino acids fermentation, were observed before 5 days of life. This was also the case for butanoic acid.

Conclusion This study shows for the first time how the metabolome changes in early life in preterm babies. A shift in the metabolome was observed after 5 days of life when the babies started to be fed and interestingly this becomes stable relatively soon (during the second week). This is likely to be related to the shift from meconium to stool. The observation of BSCFA during the first week suggests these come from the degradation of protein-rich amniotic fluid, meanwhile of acids, alcohols and esters only appears after babies were fed with milk.

\section{Colon and anorectum}

\section{CAN FIT RULE OUT COLORECTAL CANCER IN SYMPTOMATIC PATIENTS? RESULTS FROM THE NICE FIT STUDY}

${ }^{1,2}$ Nigel D'Souza, ${ }^{1,3}$ Theo Georgiou Delisle*, ${ }^{4}$ Sally Benton, ${ }^{5}$ Michelle Chen, ${ }^{1}$ Muti Abulafi. ${ }^{1}$ Croydon University Hospital; ${ }^{2}$ Basingstoke Hospital; ${ }^{3}$ Imperial College London; ${ }^{4}$ Royal Surrey County Hospital; ${ }^{5}$ Royal Marsden Partners

\subsection{6/gutjnl-2020-bsgcampus.51}

Introduction The faecal immunochemical test (FIT) is a noninvasive quantitative test which measures occult blood in faeces (faecal haemoglobin, FHb). FIT was introduced by NICE in 2017 to triage referral of patients with low risk symptoms (DG30). We report on the largest prospective and ethically approved diagnostic accuracy study to date of FIT in patients with high and low risk symptoms for colorectal cancer (CRC), meeting NICE NG12 and DG30 criteria.

Methods This multicenter study was powered to establish the diagnostic accuracy of FIT for CRC. Patients were eligible for recruitment if they experienced bowel symptoms meeting NICE two-week (TW) referral criteria and had been triaged to investigation with colonoscopy. All eligible patients referred for colonoscopy on TW pathway were asked to complete a FIT test kit (HM-JACK) prior to their colonoscopy. Patients were excluded from analysis if they did not provide a valid FIT or did not undergo complete colonoscopy. Patients were classified as high or low risk as per NICE NG12 or DG30 criteria respectively. Colonoscopy results were compared to FIT measurements of $\mathrm{FHb}$ and the conduct of the tests was double-blinded. Quality assurance of endoscopy and clinical data was performed by senior clinicians and external statisticians analysed anonymised data. This trial is registered on isrctn.com, ISRCTN49676259.

Results 9822 patients from 50 sites across England participated in the study between October 2017 to March 2019, 329 cancers were detected (3.3\% prevalence). Preliminary results for combined DG30 and NG12 groups show the sensitivity of FIT at $\mathrm{FHb}$ thresholds of 2, 10 and $150 \mu \mathrm{g} / \mathrm{g}$ was $97.0 \%$, $90.9 \%$ and $70.8 \%$ respectively. The positive predictive value (PPV) of FIT in combined groups for CRC at thresholds of 2, 10 and $150 \mu \mathrm{g} / \mathrm{g}$ was $8.7 \%, 16.1 \%$ and $31.1 \%$ respectively and the negative predictive value (NPV) of FIT at these thresholds was 99.8\%, 99.6\% and 98.9\% respectively. 6900 